\title{
miR-18a-5p promotes melanoma cell proliferation and inhibits apoptosis and autophagy by targeting EPHA7 signaling
}

\author{
YUNLONG GUO, WENLI SHI and RUIHUA FANG \\ Department of Dermatology, School of Medicine, Guangzhou First People's Hospital, \\ South China University of Technology, Guangzhou, Guangdong 510180, P.R. China
}

Received November 1, 2019; Accepted July 22, 2020

DOI: $10.3892 / \mathrm{mmr} .2020 .11717$

\begin{abstract}
Micro (mi)RNAs serve crucial roles in cancer development although little is known about their cellular mechanisms in the pathogenesis of melanoma. The present study explored the regulatory roles of miR-18a-5p in melanoma cell proliferation, apoptosis and autophagy, in addition to its target gene in melanoma cells. miRNA and ephrin receptor A7 (EPHA7) mRNA were analyzed by reverse transcription-quantitative PCR. Cell Counting Kit- 8 and colony formation assays were performed to examine the cell proliferation rate. Hoechst staining and flow cytometry were performed to investigate cell apoptosis. Western blotting was used to estimate the abundance of proteins. Dual-Luciferase reporter assay verified the binding of miRNA with target gene sequences. Melanoma tissues and cell lines exhibited markedly elevated miR-18a-5p expression. miR-18a-5p inhibitor inhibited proliferation rates, and triggered apoptosis and autophagy marker protein expression in WM266-4 and A375 cells. It also negatively regulated EPHA7 expression in WM266-4 and A375 cells by directly binding at the 3'-untranslated region of EPHA7. miR-18a-5p mimics reversed the EPHA7 overexpression-induced suppression of proliferation, and the EPHA7 overexpression-induced promotion of apoptosis and autophagy. miR-18a-5p triggered proliferation of melanoma cells and inhibited apoptosis and autophagy by directly targeting and inhibiting EPHA7 expression. Thus, the present study aided our understanding of miRNA-mediated melanoma pathogenesis.
\end{abstract}

\section{Introduction}

Melanoma is a group of severe malignant tumors that develop from transformation of melanocytes and grow on human

Correspondence to: Dr Ruihua Fang, Department of Dermatology, School of Medicine, Guangzhou First People's Hospital, South China University of Technology, 1 Panfu Road, Guangzhou, Guangdong 510180, P.R. China

E-mail: fangfrh@126.com

Key words: miR-18a-5p, ephrin receptor A7, melanoma, proliferation, apoptosis, autophagy skin, in the mouth, eyes and intestinal tissues $(1,2)$. Melanoma is the fifth and sixth most common of the human cancers affecting male and female patients respectively, according to recent epidemiological investigations. Globally new melanoma cases occur in $>0.2$ million individuals each year, an incidence that has been continuously on the rise during the past years $(3,4)$. Patients with melanoma at advanced stages who are not suitable for surgery, chemotherapy nor immunotherapy are commonly treated with drugs such as temozolomide and dacarbazine, but the efficiency of this treatment is often impaired by drug resistance $(5,6)$. The development of novel anti-melanoma drugs critically depends on the complete understanding of the molecular pathogenic mechanisms in melanoma.

EPHA7 (ephrin receptor A7) is a member of the Eph family of receptor tyrosine kinases, which are expressed in erythropoietin-producing human hepatocellular carcinoma cells (7). They are commonly known to be involved in embryonic development, angiogenesis, development of nervous and vascular systems, and a number of human pathogenic conditions, including numerous types of tumor, inflammatory diseases and neurological diseases $(8,9)$. Alteration of EPHA7 expression has been identified to be closely associated with the development of several types of human cancer over the past decade, such as colon and prostate cancer. For instance, high expression of EPHA7 is associated with adverse results among patients with primary and recurrent glioblastoma multiforme (10). By contrast, expression of the EPHA7 is markedly downregulated in colorectal cancer tissues, presumably caused by hypermethylation in the $5^{\prime} \mathrm{CpG}$ island of EPHA7 (9). EPHA7 is substantially mutated in patients with melanoma with frequent mutations recorded in tumor samples, as demonstrated by a meta-analysis of somatic mutations on the basis of next-generation sequencing (11). Nevertheless, the expressional state, regulating mechanism and functions of EPHA7 in melanoma remain to be elucidated.

MicroRNAs (miRNAs/miRs) are a large group of non-coding RNA molecules, commonly with 22 nucleotides, which target expression levels of genes by directly binding with the 3'-untranslated region (UTR) of functional genes (12). The post-transcriptional gene expression regulation by miRNAs is extensively associated with various biological processes and human disorders including several types of cancer, such as gastric, liver and breast cancer (12-14). Certain miRNAs are 
also known to modulate the function of EPHA7. For instance, miR-944 targets EPHA7 to influence the progression of lung cancer cells (15). In addition, numerous miRNAs serve crucial roles in regulating the expression of key genes associated with melanoma pathogenesis and drug responses $(16,17)$. miR-18a-5p acts has been demonstrated to be associated with cancer development and progression (18). The high levels of miR-18a-5p expression in non-small cell lung cancer (NSCLC) tissues promote proliferation and migration, and suppress apoptosis of cancerous cells by targeting interferon regulatory factor 2 gene expression (19). The expression of miR-18a-5p was reported to be markedly elevated in melanoma cell lines compared with normal human epidermal melanocytes, suggesting pathogenic roles for miR-18a-5p in the progression of melanoma (20). However, the pathogenic roles of high expression levels of miR-18a-5p in melanoma cells and the underlying molecular mechanisms, require further investigation.

The present study performed extensive analysis of miR-18a-5p expression and its cellular functions associated with melanoma development and progression using both clinical tissues and cellular models. EPHA7 was confirmed to be a target gene of miR-18a-5p in melanoma cells. The findings of the present study revealed novel molecular mechanisms through which melanoma pathogenesis is promoted, which may support miRNA-based melanoma diagnosis and treatment.

\section{Materials and methods}

Tissue collection and cell culture. Melanoma and normal skin tissues were collected from 20 patients diagnosed with malignant melanoma who underwent surgical treatment at the Department of Dermatology of the Guangzhou First People's Hospital (Guangdong, China) between July 2018 and November 2018; information about the stage and clinical status of the patient are presented in Table I. All patients provided written consent prior to the surgery and the entire study was approved by the Medical Ethics Committee of the Guangzhou First People's Hospital (approval no. K-2019-135-01). The present study was performed in accordance with the Declaration of Helsinki (21). The American Type Culture Collection supplied the normal human epidermal melanocyte PIG1 and melanoma cell lines WM266-4, A375, VMM5A and A2058. The cells were cultured in Dulbecco's modified Eagle's medium (DMEM; Thermo Fisher Scientific, Inc.) with $10 \%$ fetal bovine serum (Thermo Fisher Scientific, Inc.) and $1 \%$ penicillin or streptomycin at $37^{\circ} \mathrm{C}$ in a humidified chamber with $5 \% \mathrm{CO}_{2}$.

Reverse transcription-quantitative $(R T-q) P C R$. To examine the relative expression levels of miRNA and mRNAs, total RNA was extracted from the skin tissues or cultured cells with TRIzol ${ }^{\circledR}$ (Invitrogen; Thermo Fisher Scientific, Inc.), according to the manufacturer's protocols. A NanoDrop spectrophotometer (Thermo Fisher Scientific, Inc.) was used to record the concentration and quality of RNA. On assessing the RNA quality and concentration using a NanoDrop spectrophotometer (Thermo Fisher Scientific, Inc.), equal volumes of RNA samples $(3.0 \mu \mathrm{g})$ were collected from each group for cDNA library synthesis with the Bestar ${ }^{\mathrm{TM}}$ qPCR RT kit (cat. no. 2220; DBI Bioscience), according to the manufacturer's protocol, at $37^{\circ} \mathrm{C}$ for $1 \mathrm{~min}, 50^{\circ} \mathrm{C}$ for $60 \mathrm{~min}$ and $70^{\circ} \mathrm{C}$ for $15 \mathrm{~min}$. Then, relative levels of miRNA or mRNA were measured by RT-qPCR using the Bestar ${ }^{\mathrm{TM}}$ qPCR MasterMix kit (cat. no. 2043; DBI Bioscience), according to the following procedure: Pre-denaturation at $95^{\circ} \mathrm{C}$ for $2 \mathrm{~min}$, with 42 cycles of denaturation at $94^{\circ} \mathrm{C}$ for $20 \mathrm{sec}$; annealing at $58^{\circ} \mathrm{C}$ for $20 \mathrm{sec}$ and extension at $72^{\circ} \mathrm{C}$ for $20 \mathrm{sec}$. The maximum cycle number considered for gene expression is 42. U6 and GAPDH were used as the internal standard for quantitation of miRNA and EPHA7 expression, respectively. Expression levels were assessed by the standard $2^{-\Delta \Delta \mathrm{Cq}}$ method (22). The experiment was repeated three times. The primers used for RT-qPCR are listed in Table II.

Cell transfection. To determine the effect of miR-18a-5p expression in melanoma cells, the miR-18a-5p mimics (5'-UAA GGUGCAUCUAGUGCAGAUAG-3'), miR-18a-5p inhibitors (5'-CCAGAAGGAGCACUUAGGGCAGU-3') and negative control (NC; 5'-CAGUACUUUUGUGUAGUACAA-3') were produced and supplied by Shanghai GenePharma Co., Ltd. To determine the overexpression of EPHA7 in melanoma cells, full-length genomic sequences of EPHA7 were amplified using EPHA7-F1 (5'-CGGATCCATGGTTTTTCAAACTCGGTA CCCTTC-3') and EPHA7-R1 (5'-CCCTCGAGTCACACT TGAATGCCAGTTCCATGT-3'), which were then ligated with the pcDNA3.0 plasmid (Vipotion Biotechnology Co., Ltd.). The miRNA mimics, inhibitors and recombinant plasmids were introduced into the cultured melanoma cells using Lipofectamine ${ }^{\circledR} 2000$ transfection reagent (cat. no. 11668019; Thermo Fisher Scientific, Inc.), according to the manufacturer's protocols. The quantity of all mimics or inhibitors used was $100 \mathrm{nM}$. Alterations of miRNA and EPHA7 expression levels were confirmed by RT-qPCR following $48 \mathrm{~h}$ of transfection at $37^{\circ} \mathrm{C}$.

Colony formation assay. Melanoma cell proliferation rate was evaluated by performing a colony formation assay. Following cell transfection, WM266 and A375 cells were resuspended in DMEM and seeded in 6-well plates (100 cells/well). These were cultured under normal conditions for another 2 weeks at $37^{\circ} \mathrm{C}$. The cells were then fixed using $4 \%$ paraformaldehyde for $12 \mathrm{~min}$ at $37^{\circ} \mathrm{C}$ and stained using $0.1 \%$ crystal violet for $30 \mathrm{~min}$ at room temperature. Cell colonies were defined as $>50$ cells and counted under a fluorescent microscope (magnification, $x 400$ ) in three randomly selected fields of view for cell proliferation comparison. For statistical analysis, the experiments were performed in triplicate.

Cell counting Kit-8 (CCK-8) assay. The CCK-8 (Dojindo Molecular Technologies, Inc.) assay was performed to assess melanoma cell proliferation, according to the manufacturer's protocols. The cell suspensions (4,000 cells/well; $100 \mu \mathrm{l})$ were seeded in 96 -well plates and maintained at $37^{\circ} \mathrm{C}$ in a humidified chamber under normal conditions for 24,48 and $72 \mathrm{~h}$. Thereafter, the cells were incubated using $10 \mu \mathrm{l} \mathrm{CCK}-8$ solution for $4 \mathrm{~h}$ and absorbance measured at $450 \mathrm{~nm}\left(\mathrm{OD}_{450}\right)$ with a microplate reader. Cell proliferation was analyzed on the basis of $\mathrm{OD}_{450}$ values from $\geq 3$ replicates. 
Flow cytometry. Flow cytometry was performed to evaluate cell early apoptosis using the FITC Annexin V Apoptosis Detection kit (BioLegend, Inc.), according to the manufacturer's protocol. Melanoma cells cultured in 6-well plates were rinsed three times using cell staining buffer, resuspended in Annexin V Binding Buffer $\left(5 \times 10^{6}\right.$ cells $\left./ \mathrm{ml}\right)$ and incubated using $5 \mu 1$ FITC Annexin V and 7-AAD Viability Staining Solution for $15 \mathrm{~min}$ at room temperature in the dark. Finally, the cells were mixed with Annexin V Binding Buffer and analyzed with a FACScan flow cytometry system (BD Biosciences) and FlowJo software (version 10; FlowJo LLC). Flow cytometry analysis experiments were repeated $\geq 3$ times.

Hoechst staining. Apoptosis of WM266 and A375 cells was also evaluated using Hoechst 33258 reagents (cat. no. C1011; Beyotime Institute of Biotechnology) according to the manufacturer's protocols. Melanoma cells $\left(1 \times 10^{5}\right.$ cells/well $)$ grown on cell slides were mixed with the fixation solution at $4^{\circ} \mathrm{C}$ for $12 \mathrm{~min}$, rinsed three times using PBS, stained with Hoechst 33258 for $5-8 \mathrm{~min}$ at $37^{\circ} \mathrm{C}$ with agitation, rinsed again with PBS for 3 min at room temperature and eventually observed in three randomly selected fields of view at a wavelength of $460 \mathrm{~nm}$ using fluorescence microscopy (magnification, $x 400$ ); $\geq 3$ replicates of the experiments were performed.

Dual luciferase reporter assay. Dual-Luciferase reporter assay was performed to validate the binding of miRNA 18a-5p with the 3'-UTR region of EPHA7 in WM266 and A375 cells. The wild-type (WT) and mutated (MUT) 3'-UTR regions of EPHA7 were ligated separately with the Psi-CHECK2 plasmids (Promega Corporation), which were then transfected into WM266 and A375 cells (1x10 cells/well) using Lipofectamine 2000 as aforementioned, together with miRNA 18a-5p mimics or $\mathrm{NC}$ as designated. The cells were cultured under normal conditions at $37^{\circ} \mathrm{C}$ for $48 \mathrm{~h}$, followed by rinsing three times with PBS and incubation with passive lysis buffer. A GloMax ${ }^{\circledR}$ Bioluminescence detector (Promega Corporation) was used to measure the luciferase activity of cell lysates. The luciferase activity was normalized to Renilla luciferase activity.

Western blot analysis. Cell lysis buffer (cat. no. P0013; Beyotime Institute of Biotechnology) was used to extract total proteins from cultured cells for western blotting and immunoprecipitation according to the manufacturer's protocols. The concentration of protein was measured by a spectrophotometer at $280 \mathrm{~nm}$ using the BCA method. Total protein $(\sim 25 \mu \mathrm{g})$ collected from each group was boiled at $100^{\circ} \mathrm{C}$ for $5 \mathrm{~min}$, separated by $10 \%$ SDS-PAGE and blotted onto polyvinylidene difluoride membranes. Thereafter, the membranes were blocked with 5\% lipid-free liquid milk for 1-2 $\mathrm{h}$ at room temperature, and then incubated with primary antibodies overnight at $4^{\circ} \mathrm{C}$ and secondary antibodies for $2 \mathrm{~h}$ each at room temperature, and eventually developed with the Pierce ECL Western Blotting Substrate (Thermo Fisher Scientific, Inc.), and analyzed using ImageJ software (v1.8.0; National Institutes of Health). The following antibodies were used: anti-pro caspase-3 (1:800; cat. no. MA1-41163; Invitrogen; Thermo Fisher Scientific, Inc.), anti-pro caspase-9 (1:1,000; cat. no. MA5-32,431; Invitrogen; Thermo Fisher 
Table II. Sequences of primers used for reverse transcription-quantitative PCR.

\begin{tabular}{llr}
\hline Gene ID & \multicolumn{1}{c}{ Primer sequence $\left(5^{\prime} \rightarrow 3^{\prime}\right)$} & Product length $(\mathrm{bp})$ \\
\hline GAPDH & Forward: TGTTCGTCATGGGTGTGAAC & 154 \\
& Reverse: ATGGCATGGACTGTGGTCAT & 187 \\
EPHA7 & Forward: GTGAAGATGGGTATTACAGGGC \\
U6 & Reverse: CAACTGCACCGCTTACACAAT \\
& Forward: CTCGCTTCGGCAGCACA & 96 \\
hsa-miR-18a-5p & Reverse: AACGCTTCACGAATTTGCGT \\
& Forward: ACACTCCAGCTGGGTAAGGTGCATCTAGTGCAG & 327 \\
& Reverse: CTCAACTGGTGTCGTGGA & \\
& Reverse transcription: CTCAACTGGTGTCGTGGAGTC & \\
& GGCAATTCAGTTGAGCTATCTGC
\end{tabular}

H-/hsa, human; miR, microRNA.
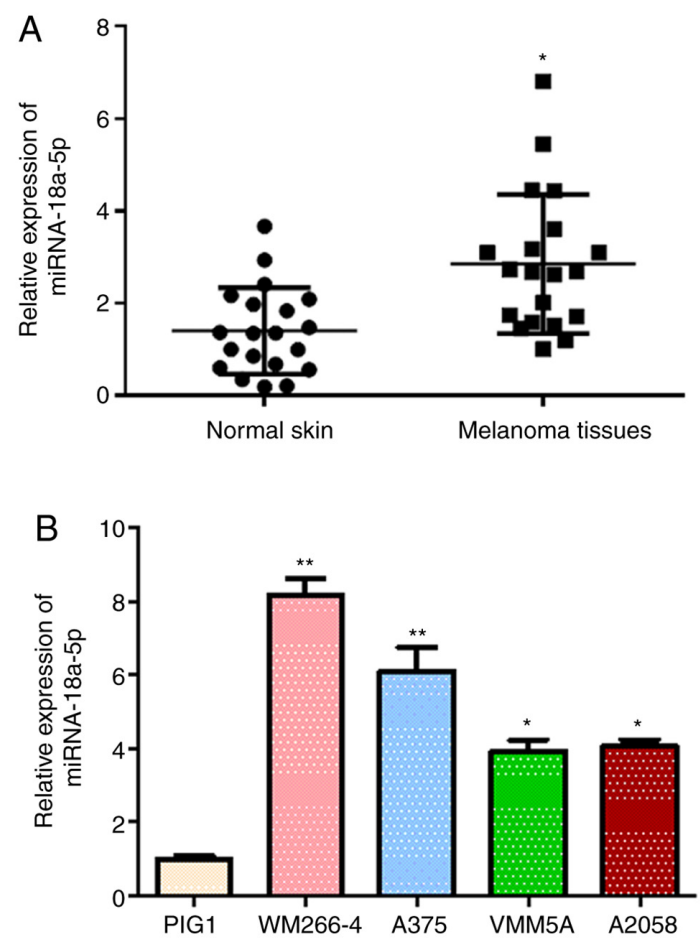

Figure 1. miR-18a-5p expression is increased in melanoma tissues and cell lines. (A) miR-18a-5p expression in melanoma tissues and adjacent normal skin tissues collected from 20 patients with melanoma. Relative expression of miR-18a-5p was determined by RT-qPCR. U6 expression was used as the internal standard. (B) Relative miR-18a-5p expression in melanoma cell lines and normal skin cells. miR-18a-5p expression in melanoma cell lines WM266-4, A375, VMM5A and A2058, in addition to normal human skin cell line PIG1, were measured by RT-qPCR. ${ }^{*} \mathrm{P}<0.05,{ }^{* *} \mathrm{P}<0.01$ vs. control. miR, microRNA; RT-qPCR, reverse transcription-quantitative PCR.

Scientific, Inc.), anti-cleaved caspase-3 (1:500; cat. no. ab2302; Abcam), anti-cleaved caspase-9 (1:500; cat. no. ab219590; Abcam), anti-autophagy marker light chain 3-I/II (LC3I/II; cat. no. 8899; CST Biological Reagents Co., Ltd.), anti-p62 (1:1,000; cat. no. ab109012; Abcam), anti-GAPDH antibodies (1:1,000; cat. no. ab9484; Abcam), goat anti-rabbit IgG (1:800; cat. no. ab205718; Abcam) and goat anti-mouse IgG (1:800; cat. no. ab205719; Abcam).
Statistical analysis. Data from $\geq 3$ replicates of all experiments are presented as the mean \pm standard deviation, and were analyzed using SPSS 20.0 (IBM Corp.). Differences between 2 groups were assessed by an unpaired Student's t-test, while differences between $\geq 2$ groups were analyzed using a one-way analysis of variance, with a Tukey's post hoc test. $\mathrm{P}<0.05$ was considered to indicate a statistically significant difference. Pearson's correlation analysis was used to analyze the correlation between miR-18a-5p and EPHA7 expression levels.

\section{Results}

Elevated miR-18a-5p expression in melanoma tissues and cells. The alterations of miR-18a-5p expression in melanoma tissues and established cell lines were identified to investigate the potential involvement of miR-18a-5p in melanoma pathogenesis. miR-18a-5p expression levels in melanoma tissues collected from 20 patients were identified to be significantly higher compared with that in the corresponding adjacent normal skin tissues (Fig. 1A). The miR-18a-5p expression levels in four human melanoma cell lines WM266-4, A375, VMM5A and A2058 were observed to be significantly elevated compared with those in the normal skin cell line PIG1 (Fig. 1B). miR-18a-5p expression levels were increased most significantly in the WM266-4 and A375 cell lines compared with the other two melanoma cell lines, so these cells were used as cellular models for the following assays. Increased miR-18a-5p expression in melanoma tissues and cell lines suggested the potential roles of miR-18a-5p during melanoma pathogenesis.

miR-18a-5p promotes proliferation and induces apoptosis and autophagy in melanoma cells. The expression of miR-18a-5p in two melanoma cell lines, WM266-4 and A375, was knocked down through transfection using specific inhibitors targeting miR-18a-5p (Fig. 2A) to investigate the cellular function of elevated miR-18a-5p expression in melanoma development. The proliferation rates of WM266-4 and A375 cells were revealed to be markedly decreased by miR-18a-5p inhibitors, compared with the NC groups, as evidenced by the 
A

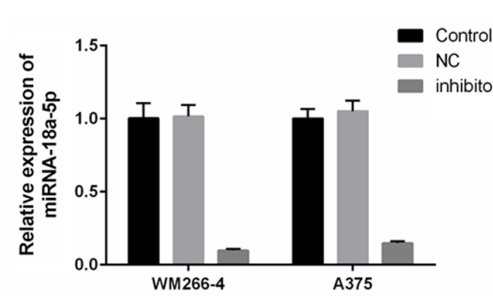

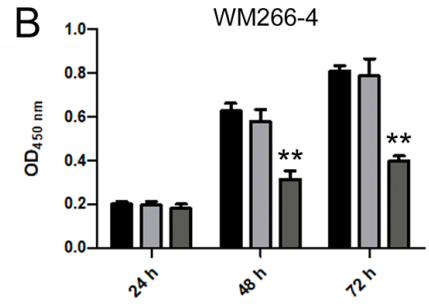

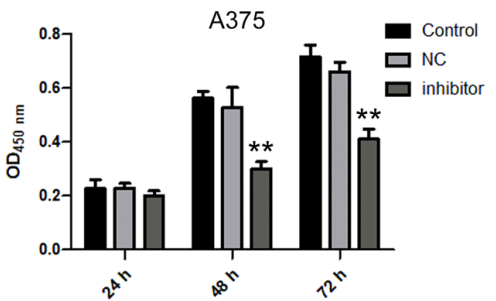

C
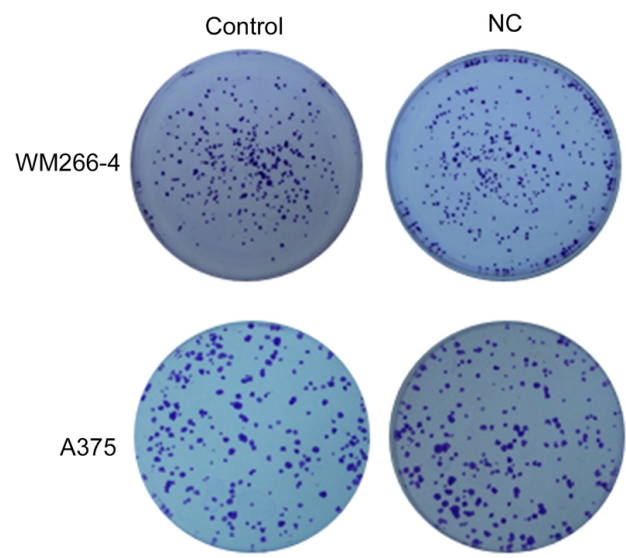

D
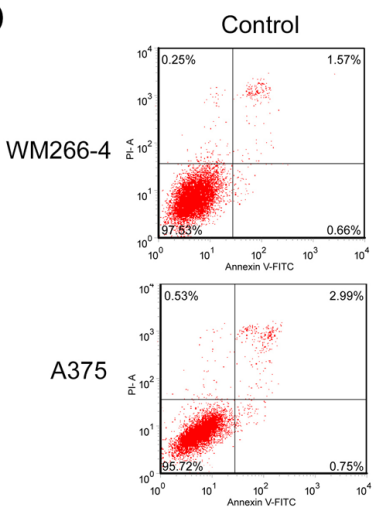

E
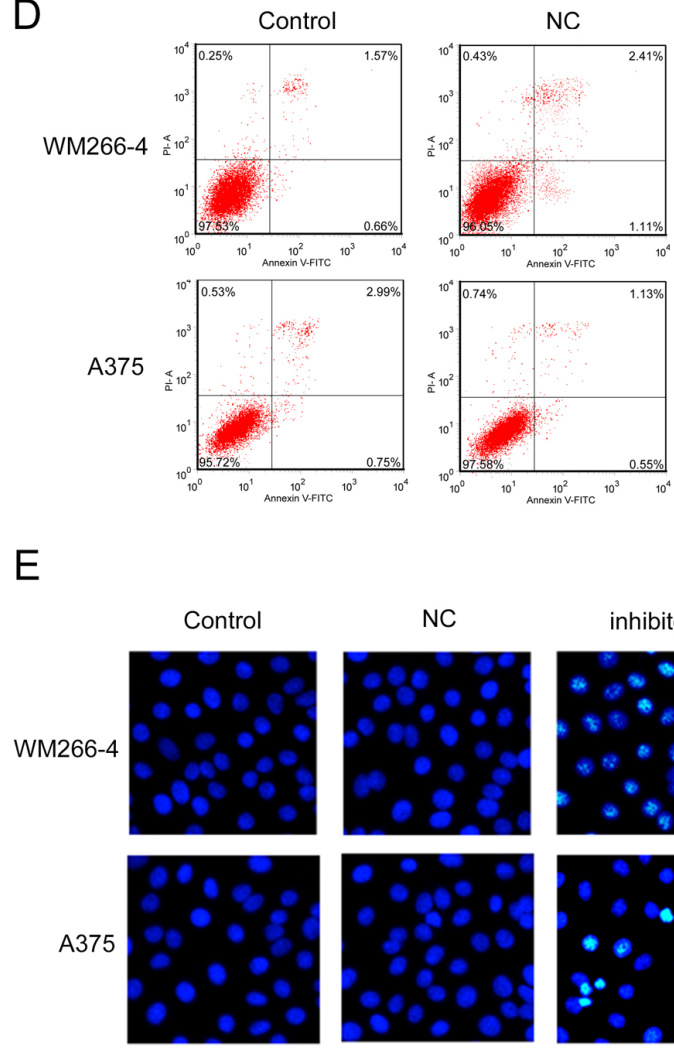

NC
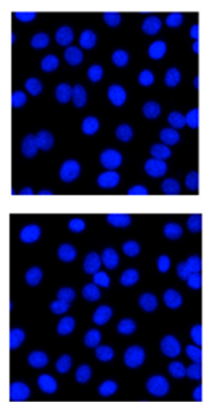
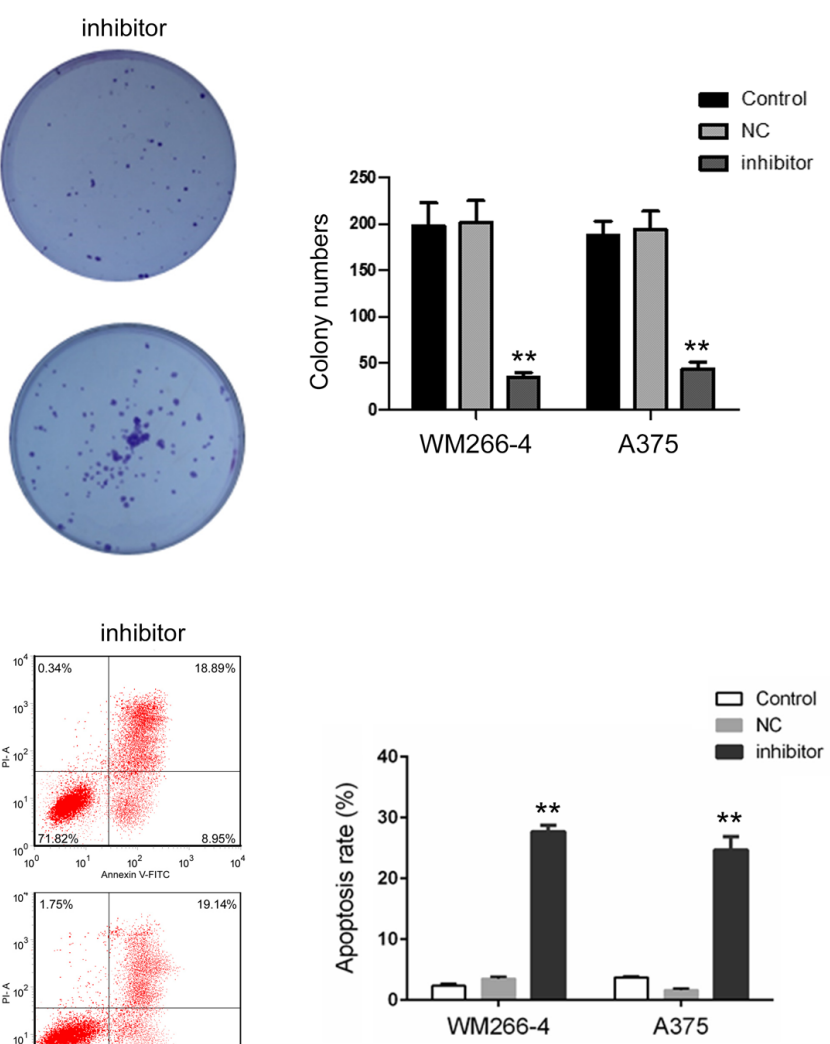

WM266-4

A375

inhibitor
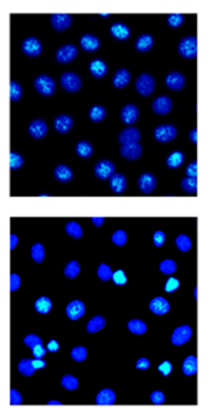

F
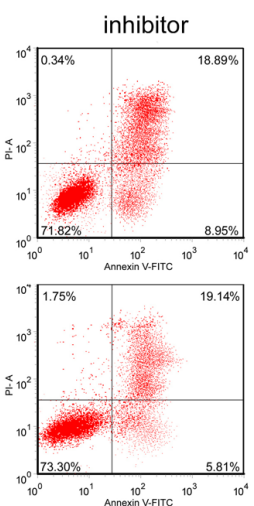

Control NC inhibitor Control NC inhibitor

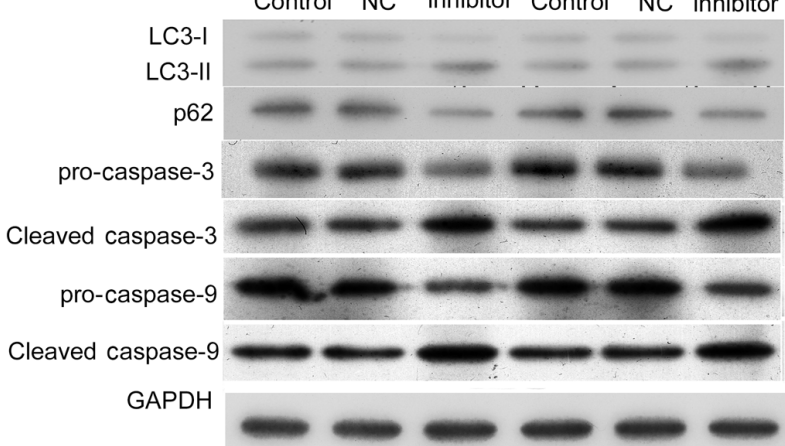

Figure 2. miR-18a-5p knockdown suppresses proliferation and promotes apoptosis and autophagy in melanoma cells. (A) miR-18a-5p expression in WM266-4 and A375 cells with specific inhibitors. miR-18a-5p expression was evaluated by RT-qPCR $36 \mathrm{~h}$ following transfection. (B and C) Decreased proliferation rates in WM266-4 and A375 cells following miR-18a-5p knockdown. Cell proliferation rates were detected by (B) Cell Counting Kit-8 and (C) colony formation assays. (D and E) Enhanced apoptosis in WM266-4 and A375 cells transfected with miR-18a-5p inhibitors. Cell apoptosis was analyzed by (D) flow cytometry and (E) Hoechst staining (magnification, x400). (F) Altered levels of apoptosis and autophagy marker proteins in WM266-4 and A375 cells following miR-18a-5p knockdown. Protein expression was measured by western blotting. GAPDH was used as the internal standard. ${ }^{* *} \mathrm{P}<0.01$ vs. NC group. miR, microRNA; RT-qPCR, reverse transcription-quantitative PCR; NC, negative control; LC3-I/II, Light chain 3-I/II.

CCK-8 assay (Fig. 2B). The colony formation assay showed a significant reduction in colony formation efficiency of WM266-4 and A375 cells induced by miR-18a-5p inhibitors
(Fig. 2C). The percentages of apoptotic WM266-4 and A375 cells were significantly increased through transfection with miR-18a-5p inhibitors in the flow cytometry assay (Fig. 2D). 

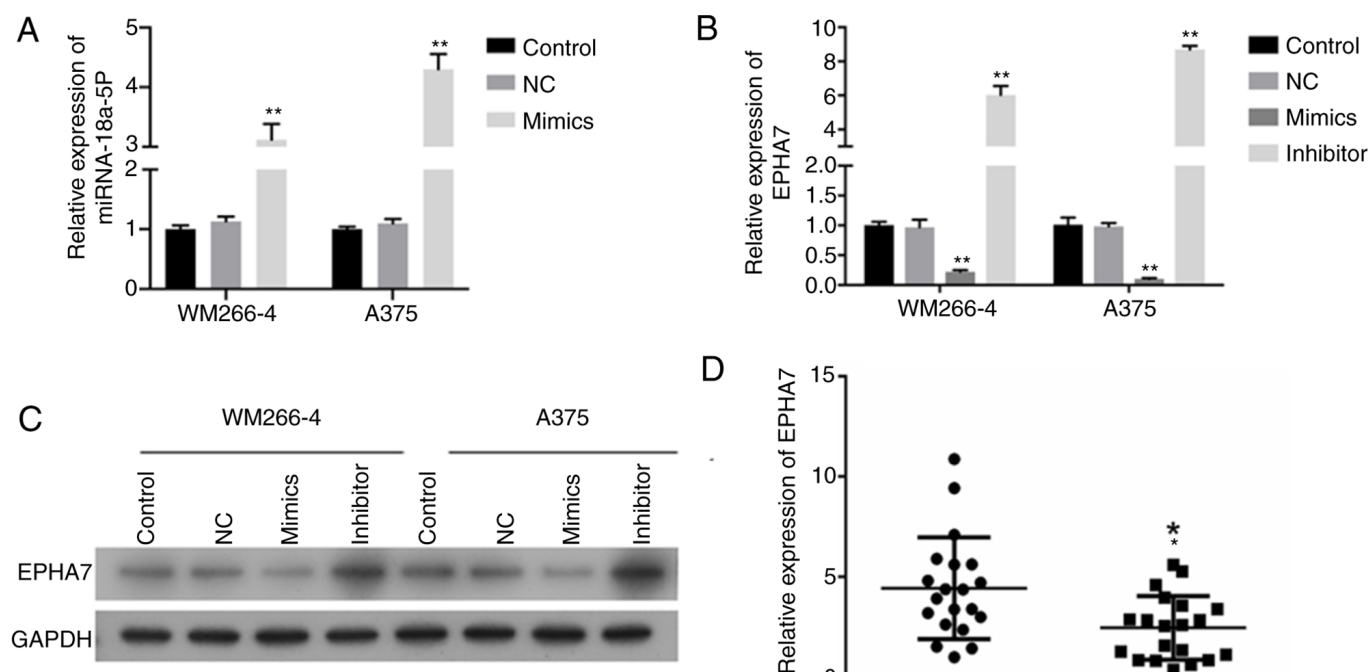

D
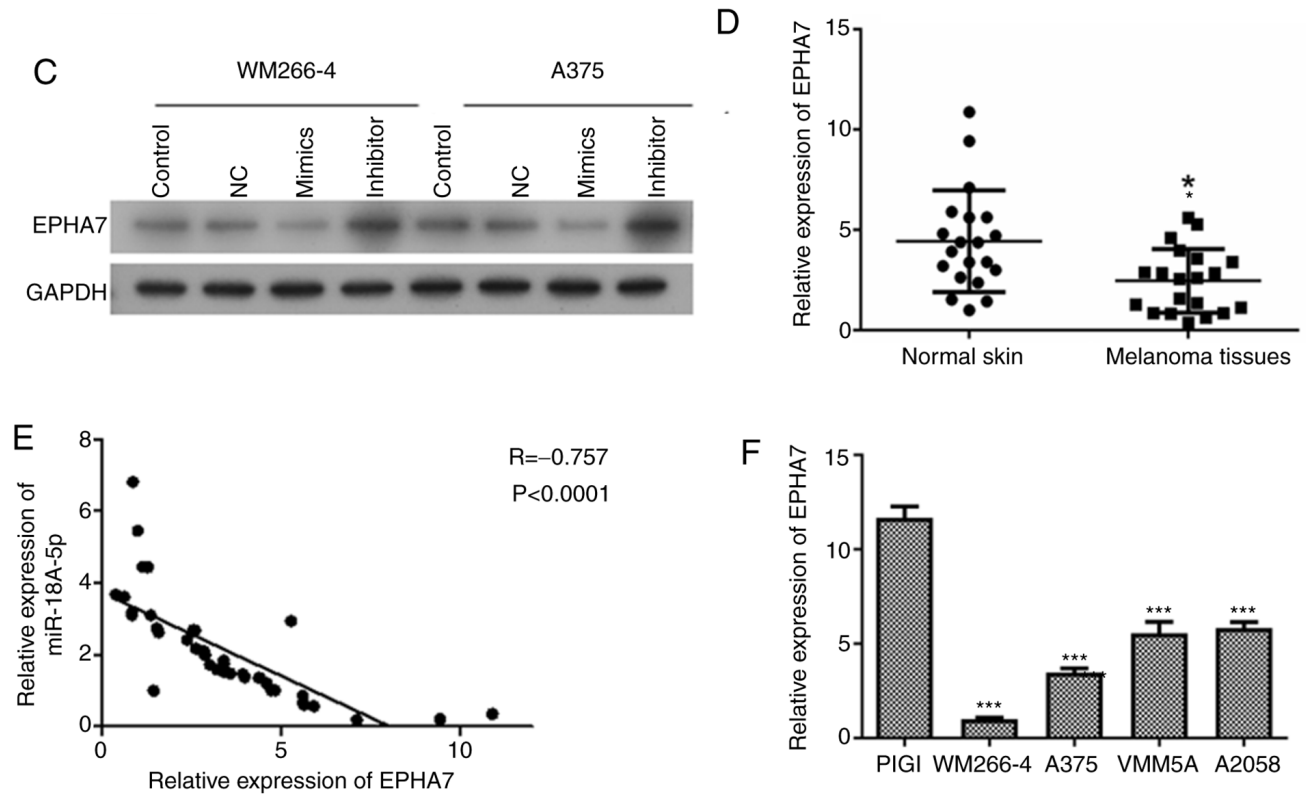

$R=-0.757$
$P<0.0001$
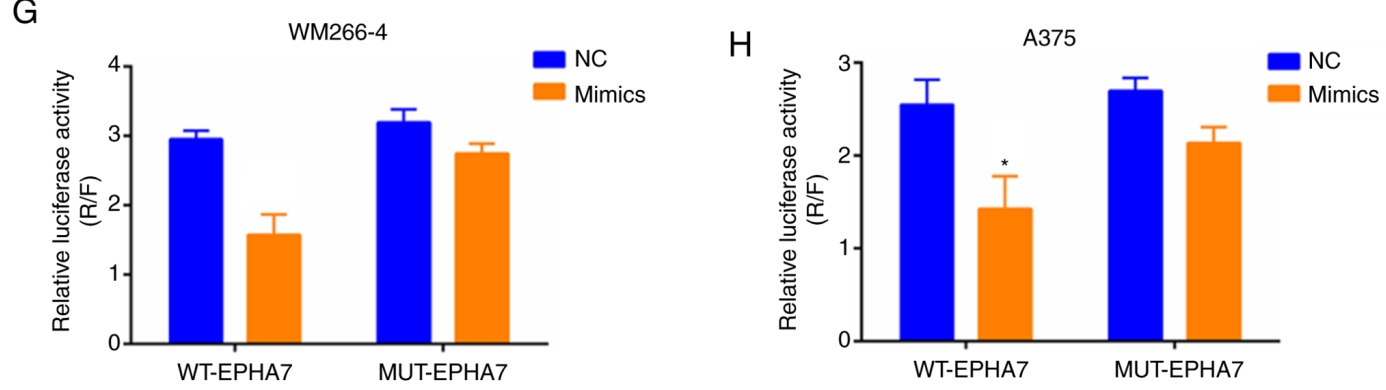

Figure 3. miR-18a-5p suppresses EPHA7 expression by binding to its 3'-UTR region in melanoma cells. (A) Relative miR-18a-5p expression levels in WM266-4 and A375 cells transfected with miR-18a-5p mimics. Gene expression was measured by RT-qPCR. (B) Relative EPHA7 expression levels in WM266-4 and A375 cells transfected with miR-18a-5p mimics or inhibitors. Gene expression was measured by RT-qPCR. (C) EPHA7 protein expression in WM266-4 and A375 cells following transfection with miR-18a-5p mimics or inhibitors. Protein expression was analyzed by western blotting, with GAPDH as the internal standard. (D) Relative EPHA7 expression in melanoma tissues and adjacent normal skin tissues collected from 20 patients. EPHA7 expression was measured by RT-qPCR. (E) Negative correlation between miR-18a-5p and EPHA7 expression in melanoma tissues and adjacent normal skin tissues. (F) Expression of EPHA7 in the indicated cell lines was examined by RT-qPCR. (G and H) Binding of miR-18a-5p with EPHA7 3'-UTR sequences in WM266-4 and A375 cells was confirmed by a Dual-Luciferase reporter assay. miR-18a-5p mimics significantly repressed luciferase activity in cells expressing WT EPHA7 3'-UTR sequences, but not in cells expressing the MUT EPHA7 3'-UTR sequences. ${ }^{*} \mathrm{P}<0.05,{ }^{* *} \mathrm{P}<0.01,{ }^{* * * *} \mathrm{P}<0.001$ vs. control group. miR, microRNA; RT-qPCR, reverse transcription-quantitative PCR; NC, negative control; EPHA7, ephrin receptor A7; UTR, untranslated region; WT, wild-type; MUT, mutant.

The Hoechst staining assay also revealed a marked elevation of apoptotic WM266-4 and A375 cells following miR-18a-5p inhibitor treatment compared with the $\mathrm{NC}$ groups (Fig. 2E). The results of western blot analysis revealed cleaved-caspase-3 and cleaved-caspase-9, the two apoptosis marker proteins, to be markedly upregulated in both WM266-4 and A375 cells following miR-18a-5p knockdown, whereas the expression levels of pro-caspase-3 and pro-caspase- 9 were notably decreased (Fig. 2F). The LC3-II protein levels in WM266-4 and A375 cells were notably elevated by transfection with miR-18a-5p inhibitors, whereas autophagy target protein $\mathrm{p} 62$ levels were markedly reduced (Fig. 2F). The aforementioned findings indicated the roles miR-18a-5p served in regulating apoptosis and autophagy during melanoma development.

miR-18a-5p suppresses EPHA7 expression by binding with its 3'-UTR region in melanoma cells. The expression of EPHA7 was further studied in melanoma cells with alterations in miR-18a-5p expression to investigate the correlation between miR-18a-5p and EPHA7 expression during melanoma pathogenesis. The expression of miR-18a-5p was significantly overexpressed following transfection with miR-18a-5p mimics (Fig. 3A). EPHA7 expression levels in WM266-4 and A375 cells were observed to be significantly suppressed by 

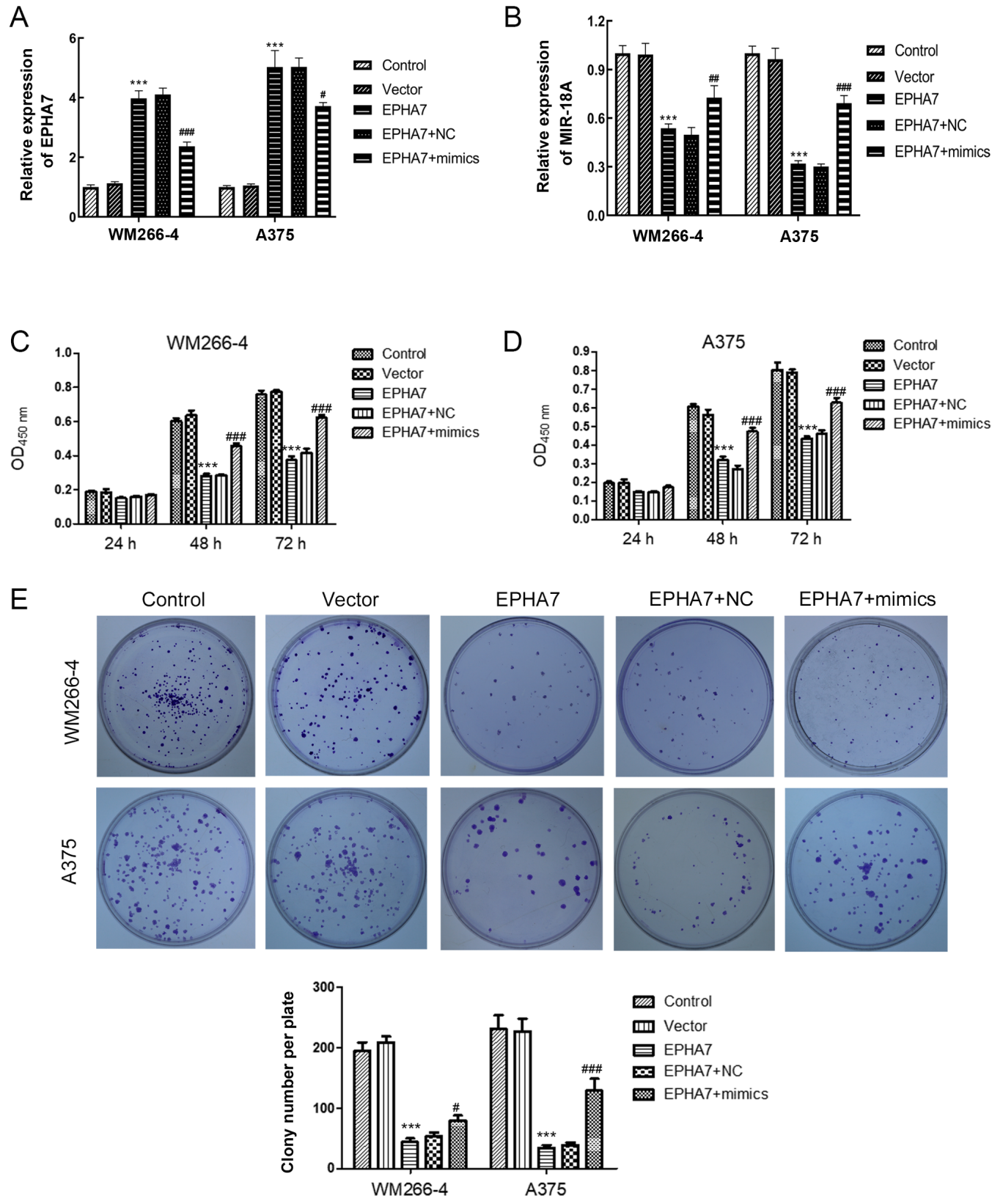

Figure 4. miR-18a-5p promotes melanoma cell proliferation by repressing EPHA7 expression. (A) Relative EPHA7 expression in WM266-4 and A375 cells transfected with EPHA7-overexpression vectors and miR-18a-5p mimics. (B) Relative miR-18a expression in WM266-4 and A375 cells transfected with EPHA7-overexpression vectors and miR-18a-5p mimics. (C) Proliferation rates of WM266-4 cells transfected with EPHA7-overexpression vectors and miR-18a-5p mimics. Cell proliferation was measured via CCK-8. (D) Proliferation rates of A375 cells transfected with EPHA7-overexpression vectors and miR-18a-5p mimics. Cell proliferation was assessed via CCK-8. (E) Evaluation of WM266-4 and A375 cell proliferation rates following transfection with EPHA7-overexpression vectors and miR-18a-5p mimics, as measured by a colony formation assay. ${ }^{* * *} \mathrm{P}<0.001$ vs. vector; ${ }^{~} \mathrm{P}<0.05$, ${ }^{\# \#} \mathrm{P}<0.01,{ }^{\# \# \#} \mathrm{P}<0.01$ vs. the EPHA7+NC group. miR, microRNA; NC, negative control; EPHA7, ephrin receptor A7; CCK-8, Cell Counting Kit-8.

transfection with miR-18a-5p mimics, but were significantly elevated by transfection with miR-18a-5p inhibitors compared with the NC groups (Fig. 3B). Western blotting results were consistent with RT-qPCR (Fig. 3C). The expression of EPHA7 in clinical melanoma tissues was also significantly lower compared with normal skin tissues (Fig. 3D). The expression of EPHA7 exhibited a significantly negative correlation with the miR-18a-5p expression in clinical melanoma tissues and normal skin tissues (Fig. 3E). In addition, EPHA7 expression levels were observed to be significantly lower in WM266-4, A375, VMM5A and A2058 cells compared with PIG1 cells (Fig. 3F). miR-18a-5p mimics were observed to cause a marked decrease in the luciferase activity of WM266-4 and A375 cells expressing the WT EPHA7 3'-UTR sequences, but not in WM266-4 and A375 cells expressing the MUT EPHA7 3'-UTR sequences (Fig. 3G and H). These findings suggested that miR-18a-5p may inhibit EPHA7 expression by directly binding with EPHA7 3'-UTR sequences in melanoma cells.

miR-18a-5p promotes melanoma cell proliferation and inhibits apoptosis and autophagy by suppressing EPHA7 expression. WM266-4 and A375 cells that overexpressed EPHA7 were established and treated with miR-18a-5p mimics to investigate the regulatory roles of EPHA7 in miR-18a-5p-promoted 
A

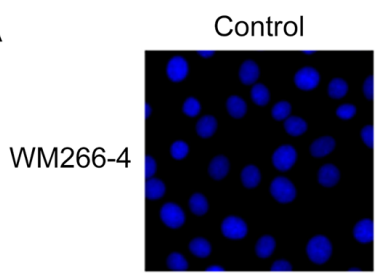

A375

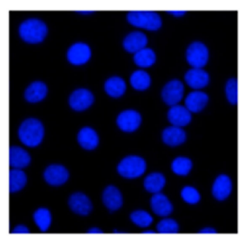

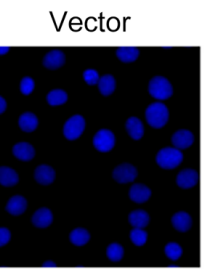

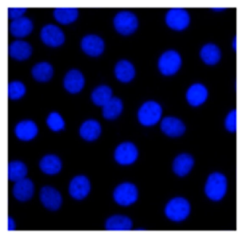

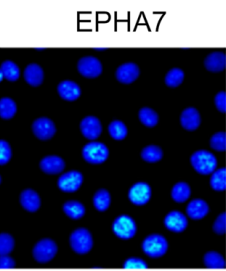

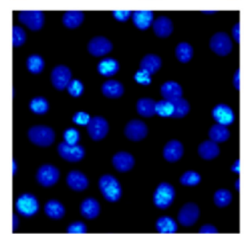

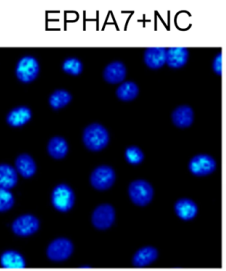

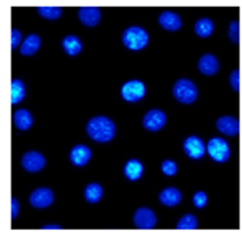

EPHA7+mimics
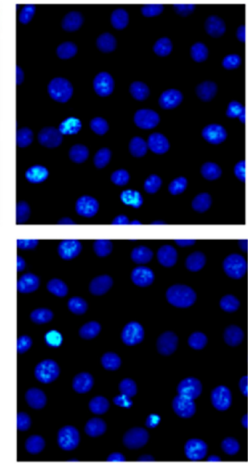

B
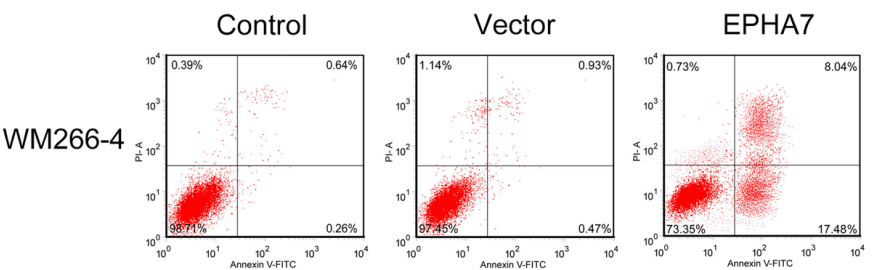

$\mathrm{EPHA} 7+\mathrm{NC}$

EPHA7+mimics
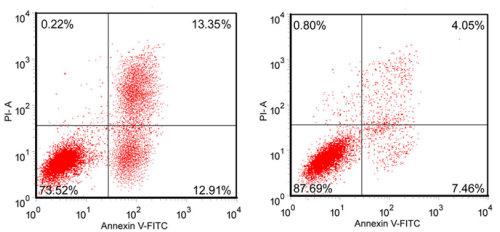

A375
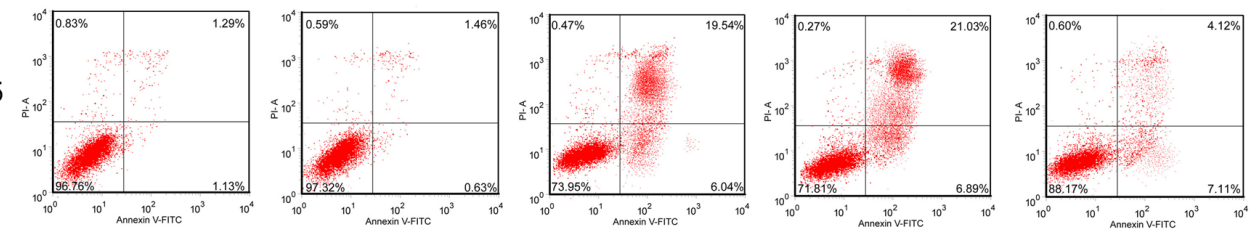

C

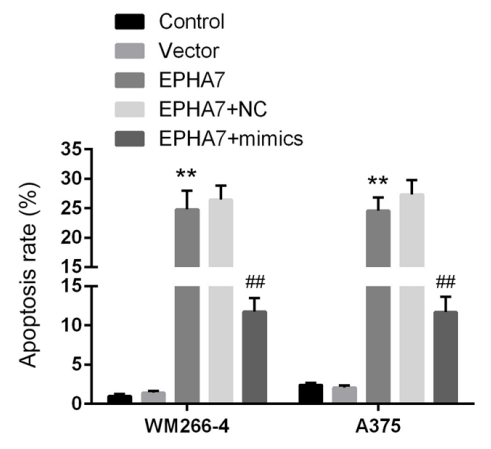

D

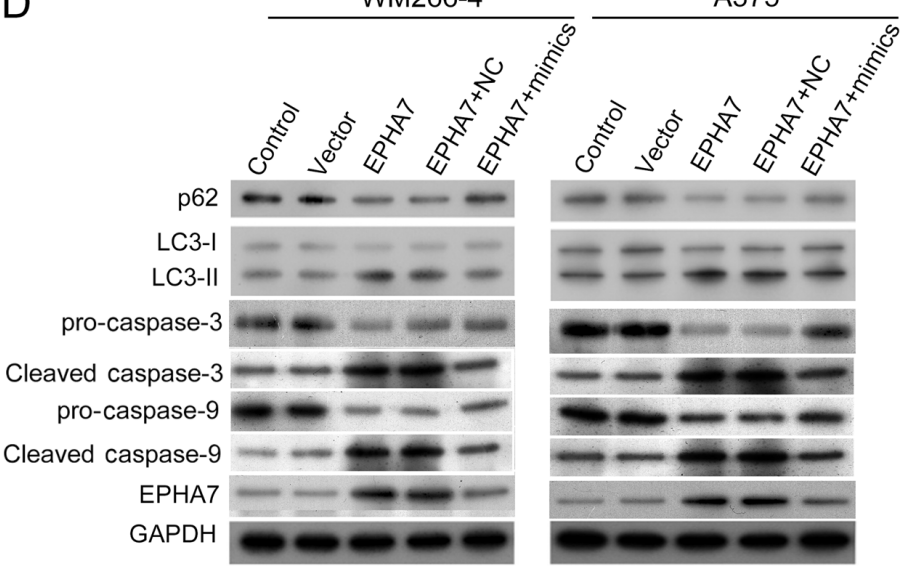

Figure 5. miR-18a-5p represses melanoma cell proliferation and autophagy by inhibiting EPHA7 expression. (A) Apoptosis of WM266-4 and A375 cells following transfection with EPHA7-overexpression vectors and miR-18a-5p mimics (magnification, x400). Cell apoptosis was detected by Hoechst staining. (B) Apoptosis of WM266-4 and A375 cells following transfection with EPHA7-overexpression vectors and miR-18a-5p mimics. Flow cytometry was used to evaluate cell apoptosis. (C) Quantitation of apoptotic WM266-4 and A375 cells following transfection with EPHA7-overexpression vectors and miR-18a-5p mimics. (D) Effects of EPHA7 overexpression and miR-18a-5p mimics on apoptosis and autophagy marker proteins in WM266-4 and A375 cells. Protein expression was determined by western blotting. GAPDH was applied as the internal standard. ${ }^{* * *} \mathrm{P}<0.01$ vs. Vector group; ${ }^{\# \# /} \mathrm{P}<0.01$ vs. EPHA7+NC group. miR, microRNA; NC, negative control; LC3-I/II, Light chain 3-I/II; EPHA7, ephrin receptor A7.

melanoma cell functions. EPHA7 expression in WM266-4 and A375 cells was observed to be significantly increased by transfection with EPHA7-overexpressing vector in the present study, but the effect of overexpression was partially inhibited by the addition of miR-18a-5p mimics (Fig. 4A). The level of miR-18a was significantly suppressed by transfection with EPHA7-overexpressing vector and this could be reversed with the addition of miR-18a-5p mimics (Fig. 4B). Overexpression of EPHA7 significantly decreased the proliferation rates of WM266-4 and A375 cells compared with the NC groups, as assessed by a CCK-8 assay (Fig. 4C). Nevertheless, the lowered cell proliferation rates in WM266-4 and A375 cells due to EPHA7 overexpression were significantly reversed by transfection with miR-18a-5p mimics (Fig. 4C and D). The colony formation assay revealed that EPHA7 overexpression led to significantly reduced numbers of WM266-4 and A375 colonies, which was significantly recovered by transfection with miR-18a-5p mimics (Fig. 4E). EPHA7 overexpression markedly increased apoptotic WM266-4 and A375 cells compared with the untransfected or empty vector-transfected cells (Fig. 5A). miR-18a-5p mimics transfection notably reduced the number of apoptotic cells (Fig. 5A). The apoptotic 
rates in WM266-4 and A375 cells were identified to be significantly elevated by EPHA7 overexpression (Fig. 5B and C). Transfection with miR-18a-5p mimics significantly eliminated the increase in apoptotic cells triggered by overexpression of EPHA7 (Fig. 5B and C). The expression levels of apoptosis marker proteins cleaved-caspase-3 and cleaved-caspase- 9 in WM266-4 and A375 cells, in addition to autophagy-related protein LC3-II, were upregulated by overexpression of EPHA7 and were inhibited by simultaneous transfection with miR-18a-5p mimics (Fig. 5D). Targets that indicate excessive autophagy degradation, p62 and LC3-I, and pro-caspase-3 and pro-caspase-9 proteins, exhibited completely opposite changes (Fig. 5D). These results indicated that miR-18a-5p could induce melanoma cell proliferation and suppress apoptosis and autophagy by inhibiting EPHA7 expression.

\section{Discussion}

Non-coding RNAs, including miRNA, long non-coding RNA and circular RNA, have been identified as crucial epigenetic regulators of various biological processes and pathogenic conditions $(23,24)$. miRNAs have also been associated with melanoma in previous studies $(16,17)$, but the precise roles and molecular mechanisms of miRNAs in the initiation and development of melanoma requires further study. miR-18a-5p promotes proliferation and migration, and suppresses apoptosis of lung cancer cells, and is also significantly increased in malignant melanoma tissues $(17,19)$; however, its pathogenic roles and mechanisms in melanoma cells remains to be elucidated. In the present study, significantly increased miR-18a-5p expression levels were recorded in clinical melanoma tissues and cell lines. By eliminating miR-18a-5p expression in melanoma cells, miR-18a-5p was demonstrated to promote melanoma cell proliferation and suppress apoptosis and autophagy in melanoma cells. miR-18a-5p was also shown to directly bind with the 3'-UTR region of the EPHA7 gene and suppress its expression in melanoma cells, which mediated the effects of miR-18a-5p expression on melanoma cell proliferation, apoptosis and autophagy. These results revealed a novel miRNA-target network underlying the pathogenesis of melanoma, which may be explored further for non-coding RNA-based melanoma diagnosis and treatment.

On the basis of previous studies, miR-18a-5p has been demonstrated to serve as a significant epigenetic regulator in various types of human cancer, including NSCLC (19), and breast $(25,26)$ and prostate cancers $(27)$. The regulatory functions of miR-18a-5p in melanoma cancer cell proliferation and apoptosis were demonstrated in the present study by transfection with miR-18a-5p inhibitors. miR-18a-5p knockdown also led to a notable elevation of the two apoptosis marker proteins caspase-3 and caspase-9, which serve as crucial components of the mitochondria-cytochrome $c$-caspase cascade of apoptosis processes (28). For the first time, to the best of our knowledge, the present study linked the cellular functions of miR-18a-5p to melanoma development and progression, which further established the widespread roles of miR-18a-5p in tumorigenesis and broadened the existing knowledge on melanoma molecular pathogenic mechanisms in terms of non-coding RNAs.

The pathogenic functions of miR-18a-5p in different types of cancer may be mediated by regulating distinct target genes (27). The suppressive effects of miR-18a-5p on EPHA7 expression was further validated in two melanoma cell lines in the present study. The Dual-Luciferase reporter assay also confirmed the direct binding of miR-18a-5p with the 3'-UTR region of the EPHA7 gene. The effects of EPHA7 on melanoma cell proliferation and apoptosis processes were reversed by transfection with miR-18a-5p mimics. These findings revealed EPHA7 as a target gene of miR-18a-5p in melanoma pathogenesis. Notably, the alteration of EPHA7 expression is also associated with the development of other types of human cancer, including glioblastoma multiforme, and colorectal, prostate and gastric cancers $(9,10,29-31)$. The miR-18a-5p/EPHA7 axis may also mediate the pathogenesis of the types of human cancer mentioned above as indicated by the involvement of miR-18a-5p in melanoma; this requires further investigation.

In addition, the miR-18a-5p/EPHA7 axis was established to alter the expression of two autophagy-related proteins LC3-I/II and p62 in melanoma cells. LC3II is one of the most specific autophagy biomarkers induced by autophagy-associated genes, including autophagy-related protein 3 (ATG3) and ATG7, and is known to closely bind with autophagosome membranes (32). By contrast, p62 protein is a commonly known degradation target of autophagy and the selective p62 degradation by autophagy has been observed to mediate the inhibitory roles of autophagy in tumorigenesis $(33,34)$. In the present study, LC3-II activation was demonstrated to be elevated by miR-18a-5p inhibitors, but p62 protein expression was suppressed by miR-18a-5p knockdown in melanoma cells. Similar LC3-II activation and alterations in p62 protein expression were also observed to be caused by EPHA7 overexpression. Molecular evidence suggested that the miR-18a-5p/EPHA7 axis modulated autophagy in melanoma cells, which may also be involved in miR-18a-5p-regulated melanoma development and progression. Autophagy has as established role in melanoma development and as a common response to antitumor therapies $(35,36)$. The pathogenic roles of autophagy alteration by miR-18a-5p in melanoma pathogenesis requires further investigations involving cellular and animal models. A limitation of the present study remains that the role of miR-18a-5p in melanoma needs to be validated through a xenograft tumor model. Furthermore, the therapeutic potential of miR-18a-5p inhibitors against melanoma remains to be further determined in clinical practice.

To conclude, miR-18a-5p was demonstrated to be highly expressed in melanoma tissues and cell lines, promoting proliferation, and suppressing apoptosis and autophagy of melanoma cells by directly targeting and inhibiting the expression of EPHA7. These findings clarified the development and progression of non-coding RNA-mediated melanoma, which may be explored further to design novel diagnostic methods and therapies for patients with melanoma.

\section{Acknowledgements}

Not applicable.

\section{Funding}

No funding was received. 


\section{Availability of data and materials}

The datasets used and/or analyzed during the current study are available from the corresponding author on reasonable request.

\section{Authors' contributions}

RF conceived and designed the research, drafted and revised the manuscript. YG performed the experiments. YG and WS analyzed the data and prepared the figures. RF and YG edited and revised the manuscript. All authors read and approved the final manuscript.

\section{Ethics approval and consent to participate}

The present study was approved by the Medical Ethics Committee of the Guangzhou First People's Hospital (approval no. K-2019-135-01) and all patients provided written consent prior to surgery.

\section{Patient consent for publication}

Not applicable.

\section{Competing interests}

The authors declare that they have no competing interests.

\section{References}

1. Bastian BC: The molecular pathology of melanoma: An integrated taxonomy of melanocytic neoplasia. Annu Rev Pathol 9: 239-271, 2014

2. Wahid M, Jawed A, Mandal RK, Dar SA, Akhter N, Somvanshi P, Khan F, Lohani M, Areeshi MY and Haque S: Recent developments and obstacles in the treatment of melanoma with BRAF and MEK inhibitors. Crit Rev Oncol Hematol 125: 84-88, 2018.

3. Ankeny JS, Labadie B, Luke J, Hsueh E, Messina J and Zager JS: Review of diagnostic, prognostic, and predictive biomarkers in melanoma. Clin Exp Metastasis 35: 487-493, 2018.

4. Siegel RL, Miller KD and Jemal A: Cancer statistics, 2018. CA Cancer J Clin 68: 7-30, 2018.

5. Corrie PG, Marshall A, Dunn JA, Middleton MR, Nathan PD, Gore M, Davidson N, Nicholson S, Kelly CG, Marples M, et al: Adjuvant bevacizumab in patients with melanoma at high risk of recurrence (AVAST-M): Preplanned interim results from a multicentre, open-label, randomised controlled phase 3 study. Lancet Oncol 15: 620-630, 2014.

6. Ichihashi N and Kitajima Y: Chemotherapy induces or increases expression of multidrug resistance-associated protein in malignant melanoma cells. Br J Dermatol 144: 745-750, 2001.

7. Li S, Wu Z, Ma P, Xu Y, Chen Y, Wang H, He P, Kang Z, Yin L, Zhao Y, et al: Ligand-dependent EphA7 signaling inhibits prostate tumor growth and progression. Cell Death Dis 8: e3122, 2017.

8. Holland SJ, Gale NW, Gish GD, Roth RA, Songyang Z, Cantley LC, Henkemeyer M, Yancopoulos GD and Pawson T: Juxtamembrane tyrosine residues couple the Eph family receptor EphB2/Nuk to specific SH2 domain proteins in neuronal cells. EMBO J 16: 3877-3888, 1997.

9. Wang J, Kataoka H, Suzuki M, Sato N, Nakamura R, Tao $\mathrm{H}$, Maruyama $\mathrm{K}$, Isogaki J, Kanaoka S, Ihara M, et al: Downregulation of EphA7 by hypermethylation in colorectal cancer. Oncogene 24: 5637-5647, 2005.

10. Wang LF, Fokas E, Juricko J, You A, Rose F, Pagenstecher A, Engenhart-Cabillic R and An HX: Increased expression of EphA7 correlates with adverse outcome in primary and recurrent glioblastoma multiforme patients. BMC Cancer 8: $79,2008$.
11. Xia J, Jia P, Hutchinson KE, Dahlman KB, Johnson D, Sosman J, Pao $\mathrm{W}$ and Zhao Z: A meta-analysis of somatic mutations from next generation sequencing of 241 melanomas: A road map for the study of genes with potential clinical relevance. Mol Cancer Ther 13: 1918-198, 2014.

12. Lin S and Gregory RI: MicroRNA biogenesis pathways in cancer. Nat Rev Cancer 15: 321-333, 2015.

13. Li Y, Qiu C, Tu J, Geng B, Yang J, Jiang T and Cui Q: HMDD v2.0: A database for experimentally supported human microRNA and disease associations. Nucleic Acids Res 42 (Database Issue): D1070-D1074, 2014

14. Valastyan S, Reinhardt F, Benaich N, Calogrias D, Szász AM, Wang ZC, Brock JE, Richardson AL and Weinberg RA: Retraction notice to: A pleiotropically acting microRNA, miR-31, inhibits breast cancer metastasis. Cell 161: 417, 2015.

15. Liu M, Zhou K and Cao Y: MicroRNA-944 affects cell growth by targeting EPHA7 in non-small cell lung cancer. Int J Mol Sci 17: 1493, 2016.

16. Penna E, Orso F, Cimino D, Tenaglia E, Lembo A, Quaglino E, Poliseno L, Haimovic A, Osella-Abate S, De Pittà C, et al: microRNA-214 contributes to melanoma tumour progression through suppression of TFAP2C. EMBO J 30: 1990-2007, 2011.

17. Stark MS, Klein K, Weide B, Haydu LE, Pflugfelder A, Tang YH, Palmer JM, Whiteman DC, Scolyer RA, Mann GJ, et al: The prognostic and predictive value of melanoma-related MicroRNAs using tissue and serum: A MicroRNA expression analysis. EBioMedicine 2: 671-680, 2015.

18. Chen X, Wu L, Li D, Xu Y, Zhang L, Niu K, Kong R, Gu J, Xu Z, Chen $Z$ and Sun J: Radiosensitizing effects of miR-18a-5p on lung cancer stem-like cells via downregulating both ATM and HIF-1 $\alpha$. Cancer Med 7: 3834-3847, 2018.

19. Liang C, Zhang X, Wang HM, Liu XM, Zhang XJ, Zheng B, Qian GR and Ma ZL: MicroRNA-18a-5p functions as an oncogene by directly targeting IRF2 in lung cancer. Cell Death Dis 8: e2764, 2017.

20. Linck L, Liebig J, Völler D, Eichner N, Lehmann G, Meister G and Bosserhoff A: MicroRNA-sequencing data analyzing melanoma development and progression. Exp Mol Pathol 105: 371-379, 2018.

21. World Medical Association: World medical association declaration of Helsinki: Ethical principles for medical research involving human subjects. JAMA 310: 2191-2194, 2013.

22. Livak JK and Schmittgen TD: Analysis of relative gene expression data using real-time quantitative PCR and the 2(-Delta Delta C(T)) method. Methods 25: 402-408, 2001.

23. Dawson MA and Kouzarides T: Cancer epigenetics: From mechanism to therapy. Cell 150: 12-27, 2012.

24. Kita Y, Yonemori K, Osako Y, Baba K, Mori S, Maemura K and Natsugoe S: Noncoding RNA and colorectal cancer: Its epigenetic role. J Hum Genet 62: 41-47, 2017.

25. Calvano Filho CM, Calvano-Mendes DC, Carvalho KC, Maciel GA, Ricci MD, Torres AP, Filassi JR and Baracat EC: Triple-negative and luminal A breast tumors: Differential expression of miR-18a-5p, miR-17-5p, and miR-20a-5p. Tumour Biol 35: 7733-7741, 2014.

26. Zhang N, Zhang H, Liu Y, Su P, Zhang J, Wang X, Sun M, Chen B, Zhao W, Wang L, et al: SREBP1, targeted by miR-18a-5p, modulates epithelial-mesenchymal transition in breast cancer via forming a co-repressor complex with Snail and HDAC1/2. Cell Death Differ 26: 843-859, 2019.

27. Zhang G, Han G, Zhang X, Yu Q, Li Z, Li Z and Li J: Long non-coding RNA FENDRR reduces prostate cancer malignancy by competitively binding miR-18a-5p with RUNX1. Biomarkers 23: 435-445, 2018.

28. Burgess JT, Bolderson E, Adams MN, Baird AM, Zhang SD, Gately KA, Umezawa K, O'Byrne KJ and Richard DJ: Activation and cleavage of SASH1 by caspase-3 mediates an apoptotic response. Cell Death Dis 7: e2469, 2016.

29. Guan M, Xu C, Zhang F and Ye C: Aberrant methylation of EphA7 in human prostate cancer and its relation to clinicopathologic features. Int J Cancer 124: 88-94, 2009.

30. Oricchio $E$ and Wendel HG: Mining the cancer genome uncovers therapeutic activity of EphA7 against lymphoma. Cell Cycle 11: 1076-1080, 2012

31. Wang J, Li G, Ma H, Bao Y, Wang X, Zhou H, Sheng Z, Sugimura H, Jin J and Zhou X: Differential expression of EphA7 receptor tyrosine kinase in gastric carcinoma. Hum Pathol 38: 1649-1656, 2007. 
32. Li MY, Zhu XL, Zhao BX, Shi L, Wang W, Hu W, Qin SL, Chen BH, Zhou PH, Qiu B, et al: Adrenomedullin alleviates the pyroptosis of leydig cells by promoting autophagy via the ROS-AMPK-mTOR axis. Cell Death Dis 10: 489, 2019.

33. Komatsu M and Ichimura Y: Physiological significance of selective degradation of p62 by autophagy. FEBS Lett 584: 1374-1378, 2010.

34. Mathew R, Karp CM, Beaudoin B, Vuong N, Chen G, Chen HY, Bray K, Reddy A, Bhanot G, Gelinas C, et al: Autophagy suppresses tumorigenesis through elimination of p62. Cell 137: 1062-1075, 2009.
35. Li Z, Jiang K, Zhu X, Lin G, Song F, Zhao Y, Piao Y, Liu J, Cheng W, Bi X, et al: Encorafenib (LGX818), a potent BRAF inhibitor, induces senescence accompanied by autophagy in BRAFV600E melanoma cells. Cancer Lett 370: 332-344, 2016.

36. Meng XX, Yao M, Zhang XD, Xu HX and Dong Q: ER stress-induced autophagy in melanoma. Clin Exp Pharmacol Physiol 42: 811-816, 2015.

(c) (i) $(9$ This work is licensed under a Creative Commons EY NG ND Attribution-NonCommercial-NoDerivatives 4.0 International (CC BY-NC-ND 4.0) License. 